(6) OPEN ACCESS

\title{
Review of the role of NICE in promoting the adoption of innovative cardiac technologies
}

Peter H Groves, ${ }^{1}$ Chris Pomfrett, ${ }^{2}$ Mirella Marlow ${ }^{3}$

${ }^{1}$ Chair, Medical Technologies Advisory Committee, National Institute for Health and Care Excellence, Manchester, UK ${ }^{2}$ Medical Technology Evaluation Programme, Centre for Health Technology Evaluation, National Institute for Health and Care Excellence, Manchester, UK ${ }^{3}$ Centre for Health Technology Evaluation, National Institute for Health and Care Excellence, London, UK

\section{Correspondence to} Dr Peter H Groves, Chair, Medical Technologies Advisory Committee, National Institute for Health and Care Excellence, Manchester M1 4BT, UK; peter. groves@nice.org.uk

Revised 18 April 2018

Published Online First 17 May 2018
Check for updates

To cite: Groves $\mathrm{PH}$ Pomfrett C, Marlow M. Heart 2018:104:1817-1822.

\section{ABSTRACT}

The National Institute for Health and Care Excellence (NICE) Medical Technologies Evaluation Programme (MTEP) promotes the adoption of innovative diagnostic and therapeutic technologies into National Health Service (NHS) clinical practice through the publication of guidance and briefing documents. Since the inception of the programme in 2009, there have been 7 medical technologiesguidance, 3 diagnostics guidance and 23 medtechinnovation briefing documents published that are relevant to the heart and circulation. Medical technologies guidance is published by NICE for selected single technologies if they offer plausible additional benefits to patients and the healthcare system. Diagnostic guidance is published for diagnostic technologies if they have the potential to improve health outcomes, but if their introduction may be associated with an increase in overall cost to the NHS. Medtechinnovation briefings provide evidence-based advice to those considering the implementation of new medical devices or diagnostic technologies. This review provides reference to all of the guidance and briefing medical technology documents that NICE has published that are relevant to the heart and circulation and reflect on their diverse recommendations. The interaction of MTEP with other NICE programmes is integral to its effectiveness and the means by which consistency is ensured across the different NICE programmes is described. The importance of the input of clinical experts from the cardiovascular professional community and the engagement by NICE with cardiovascular professional societies is highlighted as being fundamental to ensuring the quality of guidance outputs as well as to promoting their implementation and adoption.

\section{INTRODUCTION}

The National Institute for Health and Care Excellence (NICE) Medical Technologies Evaluation Programme (MTEP) was established in 2009 with the intention of simplifying access and speeding up the implementation of new medical devices and diagnostic technologies as well as encouraging collaborative research into their clinical utility and healthcare system benefits. ${ }^{12}$ A number of technologies selected by MTEP for evaluation over the last 9 years have been relevant to cardiovascular clinical practice, and their guidance preparation has highlighted important clinical messages and provided challenges for the evaluative process and opportunities for programme development.

Professional societies play a vital role in notifying medical technologies to NICE for possible evaluation. Clinicians are well placed to understand where there may be uncertainties in the case for adoption of medical technologies into clinical practice. NICE selects technologies for evaluation if they offer plausible additional benefits to patients and the healthcare system. The size of the patient population and the impact of the underlying condition on quality of life or life expectancy are also considered. The cost of the technology is material to the decision to produce guidance to avoid the National Health Service (NHS) investing in technologies that are not cost-effective. There is no defined evidentiary threshold for a technology to be selected for NICE guidance, but there must be sufficient evidence to substantiate the claimed benefits. ${ }^{3}$ In order to enhance the relevance of its medtech guidance and to address areas of unmet need, NICE is developing its topic selection function further by introducing a new entity known as MedTechScan, which will be launched in autumn 2018 .

If a technology is selected, the optimal route of assessment is chosen from the different NICE programmes to ensure that guidance is appropriate for the value proposition offered by the technology and the evidence available. For example, if a technology is likely to offer clinical benefit but is cost incurring, evaluation may be undertaken using an assessment of relative cost-effectiveness by the Diagnostics Assessment Committee or Technology Appraisal (TA) Programme. An understanding of the different NICE programmes is helpful to appreciate the context and purpose of different NICE guidance documents (table 1).

Medical technologies guidance (MTG) is prepared by the Medical Technologies Advisory Committee (MTAC), which evaluates clinical and cost benefits, the latter assessed using cost minimisation methodology from the perspective of the whole healthcare system. The MTAC processes address well-recognised challenges associated with medical device assessments. The evidence base available is often limited so that MTAC adopts a permissive approach that allows the consideration of unpublished as well as published information. Technical considerations are often important so that clinical evidence may be supported by technical evaluations undertaken by the manufacturers or by independent external assessment centres. Since the performance of medical devices is variably linked to the skill of the operator as well as to technical factors, NICE depends heavily on advice provided by experts to understand this relationship as well as to resolve uncertainties that often emerge around conflicting evidence. After consideration of all the sources of evidence, the case for adoption is supported if there is sufficient evidence to indicate superior clinical efficacy at an equivalent or reduced cost, or equivalent clinical efficacy in the face of cost savings. Partial support for adoption may result from the presence of equivocal evidence of clinical superiority or cost 


\begin{tabular}{|c|c|c|c|c|}
\hline NICE programme & Guidance considerations & Guidance recommendations & Cost analysis & Funding mandate \\
\hline $\begin{array}{l}\text { Medical Technologies Evaluation } \\
\text { Programme }\end{array}$ & Single technology & Patient and system benefits & Cost minimisation & None \\
\hline Interventional Procedures & Medical procedure & Procedural safety and efficacy & None & None \\
\hline Diagnostics Assessment Programme & Diagnostic technologies & $\begin{array}{l}\text { Diagnostic clinical and cost- } \\
\text { effectiveness }\end{array}$ & Cost-effectiveness & None \\
\hline Technology Appraisals & $\begin{array}{l}\text { Pharmaceutical or medical device } \\
\text { technologies }\end{array}$ & Clinical and cost-effectiveness & Cost-effectiveness & $\begin{array}{l}\text { NHS funding in England and Wales } \\
\text { within } 3 \text { months of publication }\end{array}$ \\
\hline $\begin{array}{l}\text { NICE Guidelines/Clinical Guidelines before } \\
\text { January } 2015\end{array}$ & $\begin{array}{l}\text { Clinical or social conditions and } \\
\text { care pathways }\end{array}$ & $\begin{array}{l}\text { Optimal clinical and social } \\
\text { practice }\end{array}$ & Cost utility & None \\
\hline
\end{tabular}

NHS, National Health Service; NICE, National Institute for Health and Care Excellence.

saving and recommendations may also encourage research into important areas of residual uncertainty. There have been 7 out of a total of 33 MTGs published for technologies that are relevant to the heart or circulation (table 2).

MTG1 supported the adoption of the SeQuent Please balloon catheter for the treatment of in-stent restenosis (ISR) having concluded that the technology was clinically superior to paclitaxel-eluting stent implantation in reducing the need for reintervention. The guidance also acknowledged, however, that further research was required to compare SeQuent Please with other drug-eluting balloon catheter and stents and recommended that studies be undertaken to include longer term clinical outcomes. The management of ISR has changed significantly since MTG1 was published in $2010,{ }^{4}$ and paclitaxel-eluting stents are no longer used in routine clinical practice. An updated evidence review was therefore recently undertaken that included an assessment of the SeQuent Please balloon catheter compared with comparators that are more relevant to current practice than paclitaxel-eluting stents. This was the first opportunity for MTEP to exercise its ability to update published MTG in the face of a substantially new evidence base, which is a common feature of innovative technologies. There were 37 studies identified that offered new information about the role of SeQuent Please in the management of ISR, including 2 systematic reviews and meta-analyses and 17 reports of randomised controlled trials. These are summarised in supporting documents considered by MTAC that are on the NICE website. It was concluded that the SeQuent Please balloon is now established in managing ISR alongside other drug-eluting balloons and second-generation drug-eluting stents. The expansion in the evidence base that was recommended in the original guidance has come to fruition, with the publication of new well-planned and executed clinical studies. The objectives of the original MTG1 to promote the adoption of, and further research into, a promising medical technology have been achieved, and consideration can now be given by NICE and other professional bodies for the inclusion of this technology in relevant clinical guidelines.

MTG32 supported the adoption of HeartFlow FFRCT for estimating fractional flow reserve from coronary CT angiography in patients with chest pain of possible ischaemic aetiology. It concluded that when used in conjunction with 64-slice (or above) CT coronary angiography imaging, the addition of this coronary physiology simulation software achieves a high level of diagnostic accuracy that means that other non-invasive testing, invasive coronary angiography and revascularisation can be avoided in some patients. These potential clinical benefits are accompanied by anticipated cost savings if high-quality CT angiographic facilities are already available. Following NICE's recommendation, HeartFlow FFRCT has been endorsed by NHS England for fast-tracking into the NHS using the Innovation and
Technology Payment programme, with the 15 Academic Health Science Networks across England taking responsibility for local adoption.

MTG33 supported the use of ENDURALIFE-powered cardiac resynchronisation therapy-defibrillator (CRT-D) devices in patients with heart failure when implantation followed the recommendations of TA 314 on implantable cardioverter defibrillators and cardiac resynchronisation therapy for arrhythmias and heart failure published in June 2014. This recommendation was made on the basis of the published evidence that indicated clinical and system benefits of extended CRT-D battery life in reducing replacement procedures. The guidance acknowledged that other competing technologies may offer similar advantages and that advances in CRT-D technology other than battery chemistry have the potential to reduce battery depletion. Nonetheless, the guidance emphasised the importance of publishing clinical outcome studies in patients receiving CRT-Ds made by all manufacturers. This would provide insights into the longevity of different CRT-Ds in real-world UK clinical practice. In this regard, MTAC was informed of a large volume of data possessed by the National Institute for Cardiovascular Outcomes Research relating to CRT-Ds implanted in the NHS since 2008 and proposed that analysis and publication of these data may provide valuable information about the longevity of different devices.

\section{MEDTECH INNOVATION BRIEFINGS (MIBS)}

MIBS are published by NICE to provide evidence-based advice to those considering the implementation of new medical devices or diagnostic technologies. Out of a total of 132 published, 23 relate to the heart or circulation (table 3 ).

The MIBs are commissioned by NHS England to support a strategy for adopting innovative medical technologies and are authored with the input of specialist commentators with knowledge of the technologies and their impact on patient care. The MIBs summarise the technology as well as describe its use in clinical practice. A summary of the relative merits of the clinical evidence and the costs associated with use of the technology is provided, but MIBs do not constitute NICE guidance and do not contain judgements or recommendations about the use of the technology in clinical practice. The intention is to provide information about innovative technologies that will assist decision making and avoid duplication, but the content may also be of interest to patients and members of the public. Technologies are selected for MIB publication if they are novel or represent an innovative iteration of a previous technology. Selected technologies are usually at a relatively early stage of evidence generation so that the preparation of an MTG would either be inadvisable or difficult. 
Table 2 A summary of NICE medical technologies guidance (MTG) relating to the heart or circulation

\begin{tabular}{|c|c|c|}
\hline MTG & $\begin{array}{l}\text { Date of } \\
\text { publication }\end{array}$ & Clinical recommendations \\
\hline $\begin{array}{l}\text { MTG1: SeQuent Please balloon } \\
\text { catheter for in-stent coronary } \\
\text { restenosis }\end{array}$ & $\begin{array}{l}\text { December } \\
2010\end{array}$ & $\begin{array}{l}1.1 \text { The case for adopting SeQuent Please balloon catheter in the NHS, when used } \\
\text { as described in } 1.2 \text { and 1.3, is supported by the evidence. The need for subsequent } \\
\text { reintervention for coronary stenosis is reduced as is the duration of clopidogrel therapy, } \\
\text { compared with paclitaxel-eluting stent... } \\
\text { 1.2 SeQuent Please balloon catheter should be considered for use in patients with in- } \\
\text { stent restenosis in bare metal coronary artery stents. } \\
1.3 \text { SeQuent Please balloon catheter can also be considered as an option for patients } \\
\text { with in-stent restenosis in any type of coronary artery stent if: } \\
\text { There are clinical reasons to minimise the duration of clopidogrel treatment (eg, } \\
\text { there is concern about an increased risk of bleeding or there is the need for surgical } \\
\text { intervention) or }\end{array}$ \\
\hline $\begin{array}{l}\text { MTG3: CardioQ-ODM } \\
\text { oesophageal Doppler monitor }\end{array}$ & March 2011 & $\begin{array}{l}1.1 \text { The case for adopting the CardioQ-ODM in the NHS, when used as described in 1.2, } \\
\text { is supported by the evidence. There is a reduction in postoperative complications, use of } \\
\text { central venous catheters and in-hospital stay (with no increase in the rate of readmission } \\
\text { or repeat surgery) compared with conventional clinical assessment with or without } \\
\text { invasive cardiovascular monitoring... } \\
1.2 \text { The CardioQ-ODM should be considered for use in patients undergoing major or } \\
\text { high-risk surgery or other surgical patients in whom a clinician would consider using } \\
\text { invasive cardiovascular monitoring. }\end{array}$ \\
\hline $\begin{array}{l}\text { MTG8: The VeriQ system for } \\
\text { assessing graft flow during } \\
\text { coronary artery bypass graft } \\
\text { surgery }\end{array}$ & $\begin{array}{l}\text { November } \\
2011\end{array}$ & $\begin{array}{l}1.1 \text { The case for adopting the VeriQ system in the NHS for assessing graft flow during } \\
\text { coronary artery bypass graft (CABG) surgery is supported by the evidence. The evidence } \\
\text { suggests that intraoperative transit time flow measurement is effective in detecting } \\
\text { imperfections that may be corrected by graft revision. This may reduce the incidence of } \\
\text { graft occlusion and may reduce perioperative morbidity and mortality. }\end{array}$ \\
\hline $\begin{array}{l}\text { MTG13: WatchBP Home A for } \\
\text { opportunistically detecting atrial } \\
\text { fibrillation during diagnosis and } \\
\text { monitoring of hypertension }\end{array}$ & January 2013 & $\begin{array}{l}\text { 1.1 The case for adopting WatchBP Home A in the NHS, for opportunistically detecting } \\
\text { asymptomatic atrial fibrillation during the measurement of blood pressure by primary } \\
\text { care professionals, is supported by the evidence. The available evidence suggests that } \\
\text { the device reliably detects atrial fibrillation and may increase the rate of detection when } \\
\text { used in primary care. This would allow prophylactic treatment to be given to reduce the } \\
\text { incidence of atrial fibrillation-related stroke. WatchBP Home A should be considered for } \\
\text { use in people with suspected hypertension and those being screened or monitored for } \\
\text { hypertension in primary care. } \\
1.2 \text { People suspected of having atrial fibrillation after use of WatchBP Home A should } \\
\text { have an ECG in line with NICE clinical guideline 36, atrial fibrillation. }\end{array}$ \\
\hline
\end{tabular}

MTG16: The E-vita open plus December 1.1 The case for adopting the E-vita open plus for treating complex aneurysms and for treating complex aneurysms $2013 \quad$ dissections of the thoracic aorta, in a carefully selected group of people, is supported by and dissections of the thoracic aorta the evidence.

1.2 Using the E-vita open plus could remove the need for a second procedure and the associated risk of serious complications, and it should therefore be considered for people:

- Who would otherwise need a two-stage repair procedure because their aortic disease extends into or beyond the distal part of their aortic arch (into the proximal descending aorta), but

- Who would not need additional intervention (such as stent grafting) in the descending aorta.

Cost consequences

1.1 ....SeQuent Please balloon catheter is associated with a cost saving of $£ 467$ per patient compared with paclitaxel-eluting stent.

MTG32: HeartFlow FFRCT for estimating fractional flow reserve from coronary CT angiography
February $\quad 1.1$ The case for adopting HeartFlow FFRCT for estimating fractional flow reserve from 2017 coronary CT angiography (CCTA) is supported by the evidence. The technology is noninvasive and safe and has a high level of diagnostic accuracy.

1.2 HeartFlow FFRCT should be considered as an option for patients with stable, recent onset chest pain who are offered CCTA as part of the NICE pathway on chest pain. Using HeartFlow FFRCT may avoid the need for invasive coronary angiography and revascularisation. For correct use, HeartFlow FFRCT requires access to 64-slice (or above) CCTA facilities.

MTG33: ENDURALIFE-powered cardiac resynchronisation therapy-defibrillator (CRT-D) devices for treating heart failure
March 2017 1.1 The case for adopting ENDURALIFE-powered CRT-D devices for treating heart failure is supported by the published evidence. Extended battery life is of clinical and patient benefit and associated with fewer replacement procedures.

1.2 ENDURALIFE-powered CRT-Ds should be considered as an option in people offered CRT-D devices in line with NICE Technology Appraisal Guidance on implantable cardioverter defibrillators and cardiac resynchronisation therapy.
11 . The cost saving per patient, when the CardioQ-ODM is used instead of a central venous catheter in the perioperative period, is about $\mathrm{f1100}$ based on a 7.5-day hospital stay.

1.2 The VeriQ system is associated with an estimated cost saving of $\mathrm{f} 115$ per patient compared with clinical assessment, when it is used routinely for assessing CABGs during surgery.

1.3 Use of WatchBP Home A in primary care is associated with estimated overall cost savings per person measured, ranging from $\mathrm{f} 2.98$ for those aged between 65 years and 74 years to $f 4.26$ for those aged 75 years and over. There is uncertainty about the costs and benefits for people younger than 65 years; however, it is plausible that using the device in this group will benefit patients and the healthcare system. Cost analyses did not support the use of the device by patients in their homes.

1.3 The E-vita open plus is estimated to generate cost savings compared with current two-stage repair from about 2 years after the procedure. The estimated cost saving per patient at 5 years afte the procedure is around $\mathrm{f} 13800$ when compared with two-stage repair involving open insertion of a vascular graft, $£ 9850$ when compared with two-stage repair involving endovascular stent grafting and $\mathrm{f} 12000$ when compared with open surgical debranching followed by endoluminal stent grafting. At 10 years after the procedure, the estimated cost savings range from around $\mathrm{f} 21850$ to $\mathrm{f} 28160$ across the three comparators.

1.3 Based on the current evidence and assuming there is access to appropriate CCTA facilities, using HeartFlow FFRCT may lead to cost savings of $£ 214$ per patient. By adopting this technology, the NHS in England may save a minimum of $\mathrm{f} 9.1$ million by 2022 through avoiding invasive investigation and treatment.

1.3 Cost modelling was based on published data using predecessor devices and showed that the price and lifespan of the CRT-D have the greatest effect on overall treatment costs. Assuming an average selling price of $\mathrm{f} 12404$ across different devices, using ENDURALIFE-powered CRT-Ds may save between $£ 2120$ and $£ 5627$ per patien over 15 years through a reduction in the need for replacement procedures. This could save the NHS in England around $\mathrm{f} 6$ million in the first 5 years.

NHS, National Health Service; NICE, National Institute for Health and Care Excellence.

\section{DIAGNOSTICS GUIDANCE (DG)}

The preparation of DG is undertaken by the Diagnostics Advisory Committee (DAC) following the assessment of single or multiple innovative diagnostic technologies or indications. Diagnostic technologies are deemed suitable for assessment by
DAC if they have the potential to improve health outcomes but if their introduction may be associated with an increase in overall cost to the NHS. The evaluation therefore addresses the evidence to support the clinical and cost-effectiveness of the technology accepting that evidence to support patient outcomes 
Table 3 A summary of NICE Medtech Innovation Briefings (MIBs) relating to the heart or circulation

\begin{tabular}{|c|c|c|}
\hline Theme & MIB & Publication date \\
\hline \multirow{3}{*}{$\begin{array}{l}\text { Coronary and peripheral } \\
\text { vascular intervention }\end{array}$} & MIB 2: The PressureWire fractional flow reserve measurement system for coronary artery disease. & February 2014 \\
\hline & MIB 55: ClearWay RX for drug delivery to coronary artery thrombotic lesions. & March 2016 \\
\hline & MIB 89: Impella 2.5 for haemodynamic support during high-risk percutaneous coronary interventions. & November 2016 \\
\hline \multirow[t]{4}{*}{ Cardiac arrhythmias } & MIB 35: AliveCor Heart Monitor and AliveECG app (Kardia Mobile) for detecting atrial fibrillation. & August 2015 \\
\hline & MIB 60: TactiCath Quartz catheter for percutaneous radiofrequency ablation in atrial fibrillation. & March 2016 \\
\hline & MIB 67: LATITUDE NXT Patient Management System for monitoring cardiac devices at home. & May 2016 \\
\hline & MIB 101: Zio Service for detecting cardiac arrhythmias. & March 2017 \\
\hline \multirow{3}{*}{$\begin{array}{l}\text { Cardiac and peripheral } \\
\text { vascular surgery }\end{array}$} & MIB 34: Spiral Flow peripheral vascular graft for treating peripheral arterial disease. & July 2015 \\
\hline & MIB 42: PROPATEN heparin-bonded vascular graft for peripheral arterial disease. & October 2015 \\
\hline & MIB 86: OCS Heart system for heart transplant. & November 2016 \\
\hline Cardiac imaging & MIB 54: Somatom Definition Edge CT scanner for imaging coronary artery disease in adults in whom imaging is difficult. & March 2016 \\
\hline \multirow{4}{*}{$\begin{array}{l}\text { Cardiopulmonary } \\
\text { resuscitation }\end{array}$} & MIB 4: The RhinoChill intranasal cooling system for reducing temperature after cardiac arrest. & February 2014 \\
\hline & MIB 18 The AutoPulse non-invasive cardiac support pump for cardiopulmonary resuscitation. & February 2015 \\
\hline & MIB 37: Thermogard XP for therapeutic hypothermia after cardiac arrest. & September 2015 \\
\hline & MIB 112: Arctic Sun 5000 for therapeutic hypothermia after cardiac arrest. & July 2017 \\
\hline Heart failure & MIB 92: CentriMag for heart failure. & January 2017 \\
\hline
\end{tabular}

NICE, National Institute for Health and Care Excellence.

for diagnostic technologies is often lower in quantity and quality than for pharmacological products because of the relatively low regulatory requirements. Out of 31 DGs published, three relate to the heart or circulation (table 4).

DG3 recommended the use of four new generation cardiac CT scanners (Acquilion OVE, Brilliance iCT, Discovery CT750 HD and Somatom Definition Flash) as options for coronary imaging in people with suspected coronary artery disease (CAD) or to determine disease progression or the need for revascularisation in people with known CAD. This guidance is particularly relevant to patients in whom imaging with earlier generation CT scanners is difficult. The new generation scanners possess enhancements as compared with their predecessors that include better temporal and/or spatial resolution and shorter acquisition times. The scope of the evaluation was limited to scanners that, although technically different, were considered to be broadly comparable for cardiac imaging. This guidance was first published in January 2012 and was updated in July 2017 and provides helpful advice to providers in planning chest pain and cardiac imaging services. This is timely in view of the prominent role proposed by NICE CG95 for CT coronary angiography in the diagnosis or exclusion of atheromatous CAD in patients presenting with chest pain.
DG15 recommended the use of two high-sensitivity troponin assays (The Elecsys Troponin T and ACHITECT STAT) for the early rule-out of non-ST-segment elevation myocardial infarction (NSTEMI) in people presenting with chest pain and suspected acute coronary syndrome. The two assays are recommended for use with early 'rule-out' protocols that typically require blood sampling at presentation and 3 hours late. This guidance emphasised the importance of interpreting the results in the context of overall clinical circumstances. Important factors highlighted include an understanding of the pretest probability of NSTEMI, the length of time since the suspected acute coronary syndrome, the possibility of chronically elevated troponin levels in some patients and the fact that the 99th percentile thresholds for troponin I and T may differ between the sexes. DG15 was published in October 2014, and the recommendations were adopted in CG95 on the assessment and diagnosis of chest pain of recent onset when it was updated in November 2016. The conclusions of DG15 supported a change in clinical practice that has subsequently been adopted more widely for the 'rule-out' of myocardial infarction in patients presenting with chest pain. ${ }^{5}$ In addition to providing clinical recommendations, DG15 described the laboratory characteristics of the two assays in detail, which will support service providers in determining which assay may best suit their local requirements.

Table 4 A Summary of NICE Diagnostics Guidance (DG) relating to the heart or circulation

\begin{tabular}{lll}
\hline DG & Recommendations & Publication date \\
\hline DG3 & $\begin{array}{l}\text { New generation cardiac CT scanners (Aquilion ONE, Brilliance iCT, Discovery CT750 HD and Somatom Definition Flash) for } \\
\text { cardiac imaging in people with suspected or known coronary artery disease in whom imaging is difficult with earlier generation }\end{array}$ \\
CT scanners. & $\begin{array}{l}\text { Atrial fibrillation and heart valve disease: self-monitoring coagulation status using point of care coagulometers (the CoaguChek September 2014 } \\
\text { XS system). }\end{array}$ \\
DG14 & $\begin{array}{l}\text { Myocardial infarction (acute): early rule out using high-sensitivity troponin tests (Elecsys Troponin Thigh-sensitive, ARCHITECT } \\
\text { STAT High Sensitive Troponin-I and AccuTnl+3 assays). }\end{array}$ \\
\hline
\end{tabular}




\section{OTHER NICE PROGRAMMES IMPORTANT TO CARDIAC MEDICAL TECHNOLOGIES}

The Interventional Procedures Advisory Committee (IPAC) prepares Interventional Procedures Guidance (IPG), which summarises the safety and efficacy of innovative procedures. These are usually notified to NICE by clinicians and other healthcare professionals. The guidance does not assess the device used in the procedure, although approximately two-thirds of IPG includes a novel medical device. Procedures that are considered suitable for evaluation include those that have not yet become established or those that are established but whose safety or efficacy has been called into question. The guidance provides advice to clinicians about practical steps that should be taken to carry out the procedure safely and effectively as well as when it is reasonable to do so. The IPG therefore highlights matters relating to clinical governance, patient consent, procedural and clinical outcome data collection and provides information about the balance of risks and benefits associated with undertaking the procedure. It is not the remit of the programme to determine the cost-effectiveness of interventional procedures or to make recommendations about whether the procedure should be commissioned but the information provided will guide local decision making. Since July 2003, IPAC have published over 600 IPGs of which 73 relate to the heart or circulation, and these are on the NICE website. While it is not possible to reference all these documents in this paper, the recent IPG on transcatheter aortic valve implantation (TAVI) provides a good example. IPG 586 was published in July 2017 and concluded that the current evidence on safety and efficacy of TAVI for aortic stenosis is adequate to support its use provided that standard arrangements are in place for clinical governance, consent and audit. In keeping with other national guidelines, ${ }^{6}$ the recommendations indicated that patient selection should be carried out by a multidisciplinary team that determines the risk for each patient and the TAVI device most suitable for them. It also specified that procedural details should be entered into the UK TAVI Registry.

NICE publishes TA Guidance for single or multiple technologies, including a recently introduced fast-track appraisal for those that offer exceptional value for money. The publication of a TA follows a request from the Department of Health and potential topics for TA assessment are mostly identified by the National Institute for Health Research Innovation Observatory. Suitable technologies include those that are likely to offer health benefits or contribute to health-related government policy but where implementation is likely to be cost incurring. The appraisal comprises an evidence-based assessment of clinical and cost-effectiveness, and recommendations about adoption result from a consideration of possible health benefits as well as the opportunity costs associated with the displacement of other treatments. There have been seven TAs published for medical devices that relate to the heart or circulation (table 5).
The relatively small number of cardiac device-related TAs is reflective of the current priority of the programme to use resources most effectively in publishing guidance on pharmacological technologies where the cost implications of adoption across the NHS are usually considerably greater than for medical devices and where (because of regulatory requirements) the published evidence is often of high quantity and quality to support an assessment of comparative effectiveness. NICE Guidelines (NG) (published as Clinical Guidelines (CG) before January 2015) provide evidence-based summaries of the diagnosis and management of specific clinical conditions, clinical or social circumstances. All the guidance documents described above may be considered in the evidence base that supports NG preparation. The emphasis, however, is on the description of the whole care pathway in which medical devices and diagnostic technologies may play a part.

\section{Wider NICE considerations}

There are a number of important principles that are common to all NICE programmes. NICE recommendations are based on the best available evidence that is considered by independent multidisciplinary committees with the input of additional clinical and patient experts. Clinical experts from the cardiovascular professional community have played a pivotal role in ensuring the quality of the guidance documents summarised in this review. Engagement between NICE and the cardiovascular professional societies is integral to the success of NICE guidance preparation and implementation. Public engagement is also of the utmost importance and, to support transparency, NICE's Committee meetings are held in public, apart from the discussion elements that involve commercially sensitive or other confidential matters. Draft guidance recommendations are subject to public consultation before being finalised and published on the NICE website, alongside the evidence base supporting the guidance. The publication of guidance on medical devices also requires full engagement by NICE with the healthcare industry and individual companies. This collaboration is built on the need for mutual trust and respect.

To avoid inconsistency in its recommendations, NICE teams actively coordinate guidance on emerging technologies with guidelines on clinical conditions, and this ensures that the most up-to-date evidence is available. A good example of this is provided by the emerging role of CT coronary imaging in the assessment of patients with chest pain. The timing of the publication of MTG32 on Heartflow FFRCT in February 2017 was planned to follow the update of the NG on the assessment and diagnosis of chest pain of recent onset (CG95), which was published in November 2016. While CG95 recommended that 64-slice CT coronary angiography should be offered as the first-line investigation of patients with stable typical or atypical

Table 5 A summary of NICE Technology Appraisals (TAs) relating to the heart or circulation

\begin{tabular}{lll}
\hline TA Guidance & Recommendations & Publication date (update) \\
\hline TA 71 & Guidance on the use of coronary artery stents. & October 2003 (July 2008) \\
TA 73 & Myocardial perfusion scintigraphy for the diagnosis and management of angina and myocardial infarction. & November 2003 (July 2011) \\
TA 88 & Dual-chamber pacemakers for symptomatic bradycardia due to sick sinus syndrome and/or atrioventricular block. & February 2005 (November 2014) \\
TA 152 & Drug-eluting stents for the treatment of coronary artery disease. & July 2008 \\
TA 167 & Endovascular stent grafts for the treatment of abdominal aortic aneurysms. & February 2009 \\
TA 314 & Implantable cardioverter defibrillators and cardiac resynchronisation therapy for arrhythmias and heart failure. & June 2014 \\
TA 324 & Dual-chamber pacemakers for symptomatic bradycardia due to sick sinus syndrome without atrioventricular block. & November 2014 \\
\hline
\end{tabular}

NICE, National Institute for Health and Care Excellence. 
anginal chest pain, MTG32 recommended that the addition of HeartFlow software in analysing the images provides the opportunity for additional clinical as well as system benefits. Both the MTG and the guideline will be reviewed by NICE to track any new emerging evidence as part of a cycle that is usually 3 years in duration. NICE is able to update guidance more frequently if new evidence has a substantial impact on existing recommendations, and professional bodies and clinicians may approach NICE if they are aware of new influential evidence emerging at an earlier date than our planned review dates, which are on the website pages.

Adoption is integral to the success of published NICE guidance, and the publication of guidance documents is accompanied by a variety of tools and programmes to support implementation, all of which are on the NICE website. The NHS funding of treatments recommended by the TA programme is normally obligatory within 3 months of publication (unless specific barriers are identified) in England and Wales. None of the other guidance documents are subject to the same legal obligations. Most importantly, healthcare professionals are instrumental in ensuring that NICE recommendations are followed to facilitate the highest quality of service delivery.

Contributors All authors have made substantial contributions to the conception or design of this paper and have contributed to drafting or revising the paper critically for important intellectual content. Final approval of the version published has been given by all authors. Agreement has been reached by all authors to be accountable for all aspects of the paper to ensure that questions related to accuracy or integrity are appropriately investigated and resolved.
Funding The authors have not declared a specific grant for this research from any funding agency in the public, commercial or not-for-profit sectors.

Competing interests None declared.

Patient consent Not required.

Provenance and peer review Not commissioned; externally peer reviewed.

Open access This is an open access article distributed in accordance with the Creative Commons Attribution Non Commercial (CC BY-NC 4.0) license, which permits others to distribute, remix, adapt, build upon this work non-commercially, and license their derivative works on different terms, provided the original work is properly cited and the use is non-commercial. See: http://creativecommons.org/ licenses/by-nc/4.0/

(c) Article author(s) (or their employer(s) unless otherwise stated in the text of the article) 2018. All rights reserved. No commercial use is permitted unless otherwise expressly granted.

\section{REFERENCES}

1 Campbell B. The NICE Medical Technologies Advisory Committee and medical technologies guidance. Heart 2011;97:674-5.

2 Campbell B, Dobson L, Higgins J, et al. A new health technology assessment system for devices: the first five years. Int I Technol Assess Health Care 2017;33:19-24.

3 Campbell B, Knox P. promise and plausibility: health technology adoption decisions with limited evidence. Int J Technol Assess Health Care 2016;32:122-5.

4 Rivero F, Cuesta J, Bastante T, et al. Treatment options for stent restenosis: insights from intracoronary imaging, clinical trials, and registries. Coron Artery Dis 2017:28:507-17.

5 Boeddinghaus J, Nestelberger T, Twerenbold R, et al. Direct comparison of 4 very early rule-out strategies for acute myocardial infarction using high-sensitivity cardiac troponin I. Circulation 2017;135:1597-611.

6 Baumgartner H, Falk V, Bax JJ, et al. ESC scientific document group. 2017 ESC/ EACTS Guidelines for the management of valvular heart disease. Eur Heart J 2017;38:2739-91. 